\title{
Modeling Patient Decision-Making: The Role of Base-Rate and Anecdotal Information
}

\author{
Angela K. Freymuth ${ }^{1,3}$ and George F. Ronan ${ }^{2}$
}

\begin{abstract}
Guidelines for managing patient-physician relationships often stem from either paternalistic or shared decision-making perspectives. Despite a number of advantages shown for the shared decision-making model, questions remain as to whether lay people make the most optimal decisions about their health care. This study explored the influence of anecdotal and base-rate information on health-care decisions. Three hundred and seventeen undergraduates read two vignettes describing a fictitious disease, followed by a description of two potential treatment protocols. The comparison treatment was $50 \%$ effective and accompanied by an anecdote that described a patient whose treatment resulted in an ambiguous outcome. A second treatment was presented as $30,50,70$, or $90 \%$ effective, and accompanied by an anecdote that described a patient whose treatment resulted in a positive, ambiguous, or negative outcome. Subjects weighted anecdotal information more heavily than base-rate, or statistical, information when the anecdotal information was clearly positive or negative. Subjects presented with ambiguous anecdotal information weighed base-rate information most heavily. Implications for enhancing patient decision-making are discussed.
\end{abstract}

KEY WORDS: patient decision-making; medical decision-making; clinical decision-making; anecdote; base-rate.

\section{INTRODUCTION}

There are several models for managing patientphysician relationships. The most traditional is the paternalistic model, which is characterized by limited patient involvement in medical decision-making. In this model, the physician takes on the role of diagnosing a condition and selecting treatment options. The basic assumption is that physicians and patients share a common goal and that physicians alone have the information and experience needed to make a competent decision (Deber, 1994). With the development of information technologies, patients have in-

\footnotetext{
${ }^{1}$ Department of Psychiatry, Division of Neuropsychology, University of Michigan, Ann Arbor, Michigan.

${ }^{2}$ Department of Psychology, Central Michigan University, Mount Pleasant, Michigan.

${ }^{3}$ Correspondence should be addressed to Angela K. Freymuth, University of Michigan Medical Center, Med Inn Room C414, Box 0840, 1500 East Medical Center Drive, Ann Arbor, Michigan 48109-0840; e-mail: angif@umich.edu.
}

creased access to information regarding the diagnosis and treatment of most medical conditions. Patients are now able to use this information to confirm diagnoses, obtain second opinions, contact support groups, and aid in their medical decision-making. This implies that patient-physician relationships are changing with more patients taking an active role in making medical decisions.

Legal mandates have also contributed to the changing nature of patient-physician relationships. Informed consent laws mandate that patients consent to treatment before any treatment can be implemented. As a result, decision-making is shared at least at a minimal level as patients understand and agree to treatment.

In contrast to the paternalistic model, the shared model of patient-physician interaction is characterized by a two-way decision-making process. The shared model has been proposed as the ideal model for medical decision-making, with physicians and patients working together to craft optimal decisions 
(Deber, 1994). There are significant advantages to the shared decision-making model. For example, individuals who participate in decisions about their own medical treatment have been found to have less morbidity than those who do not participate (Greenfield, Kaplan, \& Ware, 1985; Lerman et al., 1990; Mahler \& Kulik, 1990). Increased involvement of patients in medical decision-making has also been found to increase satisfaction with medical treatment in cases where there is more than one alternative treatment recommendation (Moyer \& Solovey, 1998). Finally, shared decision-making has been found to be related to increased adherence to treatment (Speedling \& Rose, 1985).

Despite the fact that patient-physician interactions following the shared decision-making model have been found to have a positive impact, the questions remains: Can patients make accurate and effective decisions regarding their health care? What are the obstacles?

Some research suggests that even with adequate information, eliciting reasonable, informed and unbiased decisions from patients can be a difficult task (Redelmeier, Rozin, \& Kahneman, 1993). There are a number of errors that individuals make when faced with decisions in general. These include the anchoring heuristic, biased search strategies, and the availability heuristic. In a review of the medical decisionmaking literature, Redelmeier et al. (1993) outlined several decision-making errors that patients often make when faced with a medical problem. These errors can be conceptualized into three broad categories: (1) over reliance on past experiences, (2) emotional override, and (3) not comprehending statistical information.

In a classic article (Kahneman \& Tversky, 1973), Kahneman and Tversky provided participants with a personality description of an individual whose personality was similar to a stereotypical depiction of either a lawyer or an engineer. Participants were told the base-rate of lawyers to engineers was 7 to 3 . Participants subsequently guessed the occupation that went with each personality description. Base-rate information had no discernable impact. That is, personality descriptions that matched the stereotype of a lawyer were attributed to a lawyer and personality descriptions that matched the stereotype of an engineer were attributed to an engineer. The authors commented that people lack the cognitive ability to deal with baserate information. That is, subjects did not understand the robustness of statistics based on large samples and relied on individual portrayals. This blindness to sample size has also been coined the "belief in the law of small numbers" (Tversky \& Kahneman, 1971).

The authors are unaware of previous studies that have used an analogue design to explore what information people pay attention to when making medical decisions. The current study aimed to do just this. More specifically, this study asked: How do college students weight anecdotal versus base-rate, or statistical, information when making judgments in the context of medical decision-making? The ultimate goal was to generate hypotheses regarding how physicians might structure information to enhance patient decision-making.

\section{METHODS}

Participants

Three hundred and seventeen students from undergraduate psychology courses at a medium size Midwestern University participated in this study. All participants read and signed the Internal Review Board approved informed consent document prior to their participation. Ages ranged from 18 to 25 $(M=19 ; S D=1.45)$. Seventy-six percent $(n=241)$ of the participants were female. Fifty-three percent $(n=168)$ were freshmen, $24 \%(n=76)$ were sophomores, $15 \%(n=48)$ were juniors, and $8 \%(n=25)$ were seniors. Of the 312 subjects who reported their race, $89 \%$ were Caucasian.

Materials

Vignettes

A series of vignettes assessed decision-making. The vignettes described a fictitious disease, Schistomanliasis (SCIMAS). A fictitious disease was used to eliminate the possibility of familiarity with the information about the disease. The name, Schistomanliasis, was derived from a combination of diseases endemic to Africa and the parasites that cause them. Symptoms associated with Creutzfeld-Jacob's Disease were used to develop fictitious disease related symptoms. More specifically, SCIMAS was described as a disease that causes significant muscular and cognitive difficulties that, if left untreated, eventually lead to death. SCIMAS infection reportedly stemmed from exposure to the microscopic eggs of a parasitic flatworm. It was presented as affecting men and women of all ages and racial groups. To further increase the relevance, the 
disease was presented as a particular risk to individuals who reside in the Midwestern section of the United States. Finally, to ensure that participants would understand the importance of making a treatment decision, it was explicitly stated that untreated individuals would certainly die within a period of 12 months. All subjects received the exact same description of SCIMAS.

The two fictitious treatments for SCIMAS were developed in a similar manner. The name for the control treatment, Fluortrexate, was derived from a combination of two chemicals commonly used in chemotherapy, Fluorouracil and Methotrexate. The name of the manipulated treatment, Tamoxol, was derived from combining the names for two common chemotherapy drugs, Tamoxifen and Taxol. Both treatments were presented as chemical treatments, consequences presented for the procedures were equal, and the number of words used to describe each treatment was equal.

The vignette involving Fluortrexate was always presented as $50 \%$ effective and served as the comparison condition. The vignettes involving Tamoxol presented the treatment effectiveness base-rate as 30, 50,70 , or $90 \%$ effective. Except for the changing baserate, the number of words, the structure, and the content of all information regarding treatment effectiveness were identical.

Participants also read anecdotal accounts of patient responsiveness to treatment. The anecdote paired with Fluortrexate was about Chris, a 20year-old "typical" college student who hoped to be a schoolteacher someday. Chris was diagnosed with SCIMAS and was faced with choosing a treatment. Chris chose Fluortrexate and the results were ambiguous: Chris and the doctors were not sure if deciding on Fluortrexate was the right or the wrong decision.

The anecdotes paired with Tamoxol were positive, ambiguous, or negative. The story was about Pat, a 20-year-old "typical" college student who eventually wanted to teach at the college level. Pat was diagnosed with SCIMAS and was faced with choosing a treatment. The overall format for the different Tamoxol anecdotal conditions follows.

Positive anecdote condition: Pat's decision to undergo Tamoxol resulted in a positive outcome. The entire worm was destroyed. Doctors were confident the disease would not resume its course. At 1-month posttreatment Pat's recovery was certain.
Negative anecdote condition: Pat's decision to undergo Tamoxol resulted in a poor outcome. The worm was not completely destroyed. The disease resumed its course. At 1-month posttreatment Pat was blind and has lost the ability to walk.

Ambiguous anecdote condition (Tamoxol and Fluortrexate): Pat/Chris was unsure if the decision was right or wrong (i.e., was ambiguous). Doctors were not certain whether the worm was destroyed. Doctors were unable to determine whether the disease would resume its course. At 1 -month posttreatment Pat/Chris is having good days and bad.

To reduce potential bias, the content and structure of the anecdotes for the control treatment (Fluortrexate) and the manipulated treatment (Tamoxol) were very similar with only a few details changed to ensure the stories were realistic. Both the character names (Chris and Pat) and the career path (teacher and college professor) were gender-neutral. The number of words devoted to the different treatments, anecdotal information, and base-rates was identical. Finally, the order of presentation for the base-rate and anecdotal information was counterbalanced.

\section{Comprehension of Vignette Information}

A quiz assessed whether participants processed the information presented in the vignettes. To enhance motivation levels, participants were told ahead of time that they would be given snack items and candies of their choice based on their performance on the quiz. Items on the quiz were organized into three distinct groups; knowledge, believability, and understandability. The six-item knowledge measure asked questions about the name of the disease, anecdotal information, and base-rate information. Answers were scored as either correct (2) or incorrect (1). The believability scale contained three items about the disease, the treatments, and the afflicted individuals. Participants rated how believable the information was using a 10-point scale and internal consistency was adequate $(\alpha=.82)$. The understandability scale contained three items about the disease, the treatments, and the afflicted individuals. Participants rated how understandable the information was using a 10point scale and internal consistency was acceptable $(\alpha=.80)$. 
Procedure

Subjects were randomly assigned to 1 of the 12 conditions ( 4 base-rate conditions and 3 anecdote conditions). This was done by randomly ordering the 12 different versions of the information packet and handing them out to students seated in their classrooms. After reading the instructions, subjects completed a demographic information form, read descriptive information about SCIMAS, and read the control treatment vignette. To control for order effects, half of the subjects read the anecdotal information about Tamoxol and then the base-rate information about Tamoxol, whereas the other half read the base-rate information about Tamoxol and then the anecdotal information about Tamoxol. Subjects were instructed to place themselves in the situation as much as possible and to choose a course of treatment (i.e., Fluortrexate or Tamoxol). Subjects handed the materials back to the experimenter and completed the quiz about the quality of information presented. Rewards and debriefing forms were dispensed.

\section{RESULTS}

Point-biserial correlations were used to determine whether age, gender, race, year in school, order of presentation of the material, history of past illnesses, previous experience with medical decisionmaking, current perception of health, or reported prior knowledge of SCHIMAS were related to the treatment choice. The lack of any significant findings suggests that treatment choice was independent of these variables.

A two-dimensional MANOVA was used to determine whether subjects exposed to the different conditions processed the information to a similar extent. More specifically, a 3 (positive, ambiguous, or negative anecdotes $) \times 4(90,70,50$, or $30 \%$ base-rate $)$ MANOVA was used, with scores on the Believability, Knowledge, and Understandability questions serving as the dependent variables. The results showed a significant Omnibus $F$ for anecdotes, $F(6,604)=3.74$; $p<.01$. No significant differences were found for base-rate information, $F(2,303)=0.57 ; p=.82$, or the interaction between anecdote and base-rate information, $F(18,909)=1.32 ; p=.17$. Univariate analyses revealed significant differences for the anecdote condition on two of the dependent measures; Knowledge, $F(2,303)=5.82 ; p<.01$, and Understandability, $F(2,303)=5.51 ; p<.01$. Follow-up contrast using Tukey's HSD test revealed that subjects in the negative anecdote conditions obtained lower scores on the Knowledge items than subjects in either the positive $(p=.01)$ or the neutral $(p=.02)$ anecdote conditions. No other significant differences were found. On the Understandability items, the only significant difference was subjects in the negative anecdotal condition obtained lower scores on the Understandability items than subjects in the positive anecdotal condition $(p=.01)$. A perusal of the means associated with each condition revealed only small differences (Knowledge mean scores ranged from 10.60 to 11.40 ; Understandability mean scores ranged from 21.32 to 23.31 ).

Treatment Chosen

Because treatment chosen is a dichotomous variable, chi-square analyses were used to test hypotheses. Analyses were conducted with and without controlling for scores on the Knowledge and Understandability scores. Because controlling for Knowledge and Understandability scores had no significant impact, the results of the simpler (noncontrolled) analyses are presented.

An initial analysis revealed that assignment to conditions had a significant influence on treatment choice, $\chi^{2}(1,11)=117.17, \quad p<.001$. Subsequent analyses revealed a significant impact for anecdotes, $\chi^{2}(1,2)=84.56, p<.001$, base-rate, $\chi^{2}(1,11)=24.81, p<.001$, and the interaction between anecdotes and base-rate, $\chi^{2}(1,7)=96.84, p<$ .001 . Follow-up analyses contrasted the proportion of subjects who chose the manipulated treatment in the positive and negative anecdote conditions with the percentage of subjects who chose the manipulated treatment in the ambiguous condition. Additional analyses contrasted the number of subjects who chose the manipulated treatment in the 90,70 , and $30 \%$ base-rate conditions with the number of subjects who chose the manipulated treatment in the $50 \%$ base-rate condition. As shown in Table I, subjects exposed to the negative anecdote were significantly less likely to choose the manipulated treatment than subjects exposed to the ambiguous anecdote. Moreover, subjects exposed to the positive anecdote were more likely to choose the manipulated treatment even when the base-rate for that treatment was significantly less than the alternative treatment $(50 \%$ in the control treatment vs. $30 \%$ in the manipulated treatment). With regard to the influence of base-rate, participants in the negative anecdote condition were still more 
Table I. Percent of Subjects Choosing the Manipulated Treatment (Tamoxol)

\begin{tabular}{lcccc}
\hline & \multicolumn{4}{c}{ Base-rate } \\
\cline { 2 - 5 } & $90 \%$ & $70 \%$ & $50 \%$ & $30 \%$ \\
\hline Positive anecdote & 88 & 92 & $93^{a}$ & $78^{a}$ \\
$n$ & 26 & 26 & 27 & 27 \\
Ambiguous anecdote & 81 & 81 & 69 & $29^{b}$ \\
$n$ & 26 & 26 & 26 & 28 \\
Negative anecdote & $39^{a, b}$ & $43^{a, b}$ & $15^{a}$ & $07^{a}$ \\
$n$ & 28 & 24 & 26 & 27 \\
\hline
\end{tabular}

${ }^{a}$ Significantly different from the corresponding ambiguous anecdote condition $(p<.05)$.

${ }^{b}$ Significantly different from the $50 \%$ base-rate condition $(p<.05)$.

likely to choose the manipulated treatment when the base-rates were significantly higher (90 and $70 \%$ ) than the $50 \%$ base-rate.

A 3 (anecdote) $\times 4$ (base-rate) ANOVA was used to test how confident subjects were in their treatment decisions. A main effect was found for the influence of anecdotal information on confidence ratings, $F(2,304)=4.94 ; p<.01$. The main effect for base-rate information, $F(3,304)=0.396 ; p=.756$, as well as the interaction between anecdote and baserate were not significant, $F(6,304)=1.48, p=.185$. Post hoc contrasts using Tukey's HSD statistic were employed to determine where these significant differences emerged. Overall, subjects in the positive anecdote condition $(M=6.89, S D=2.23)$ reported more confidence in their decisions than subjects in the ambiguous anecdote condition $(M=5.87 ; S D=$ $2.54 ; p=.006)$. There were no significant differences for subjects in the negative anecdote condition $(M=$ $6.51, S D=2.26$ ).

\section{DISCUSSION}

This study explored how undergraduate college students weight base-rate versus anecdotal information when making judgments in a health-related context. In general, subjects weighted anecdotal information greater than base-rate information. For instance, subjects chose a less effective treatment if the more effective treatment was paired with a negative anecdote. In addition, subjects were more likely to choose a $30 \%$ effective treatment, in comparison to a $50 \%$ effective treatment, when the $30 \%$ effective treatment was paired with a positive anecdote. In contrast, subjects in the ambiguous anecdotal conditions were more likely to base their decision-making on base-rate information. That is, in the absence of clear anecdotal information decisions were more likely to match base-rate information.

Patients are exposed to a considerable amount of information about diseases and treatments through mediums such as television, the Internet, friends, and family. Most of this information is likely to be anecdotal and these results imply that such information is likely to exert a strong influence over patient decision-making. Although some research has found a shared decision model for patient-physician relationships is the most efficacious (Deber, 1994), health-care providers who espouse this model might need to be more conscious of the impact of providing anecdotal information so that the most effective treatments are heavily weighted. Choosing a treatment is likely to be enhanced by pairing the treatment with a positive anecdote. Avoiding a treatment is likely to be enhanced by pairing the treatment with a negative anecdote. Because base-rate information did influence decision-making when treatments were new and anecdotal information was scarce, a clear presentation of the ambiguous anecdotal information is likely to help patients to choose treatments based on baserate data. Finally, physicians can probably devote less attention to the order in which they present information, as it did not matter whether participants initially received anecdotal or the base-rate information.

Having subjects rate their certitude for choosing one treatment over another assessed the influence of anecdotal and base-rate information on the degree of confidence in their decision-making. Exposure to the positive anecdote, in contrast with exposure the ambiguous anecdote, resulted in higher confidence ratings. No significant difference emerged when contrasting subjects exposed to the negative anecdote with subjects exposed to either the positive or ambiguous anecdote. That is, subjects in the negative anecdote condition chose the treatment with a lower base-rate of success more often that subjects in the positive condition, but their ratings of confidence in their decisions were similar. Moreover, subjects rated negative information as slightly less understandable than the positive information and this implies that students were less likely to understand or remember negative information presented. Nonetheless, negative anecdotal information did influence treatment decisions and patient overall level of confidence in their decision-making was not influenced. There is little research available to guide speculation. These findings suggest that further research on how patients process negative information is needed. 
The use of healthy undergraduate students sets limits on the degree to which these findings can be generalized to real-life health-care decision-making. The present study was able to control for many threats to internal validity and we believe this is important because of the lack of existing research in this area. Clearly, people who are currently suffering from medical illness have a more vested interest in choosing an appropriate medical treatment and might be prone to more carefully weigh information. However, they may also be more emotionally involved and this emotionality might reduce their ability to think objectively. According to Raghunathan and Pham (1999), individuals faced with serious medical decisions are under a great deal of stress and the decision-making process could be altered by their affective state. In addition to emotionality, real-life health-care decisionmaking might also be influenced by past experiences. Most undergraduate students report little personal experience with serious illness and important medical decisions. Therefore, the sample in this study might be less prone to making health-care decisions based on anecdotal information or past experiences. We anticipate exploring these effects using clinical samples.

Another line of research could begin to examine how anecdotal and base-rate information influence the health-care decisions of people across the lifespan and, in particular, older healthy populations for whom medical decisions are more salient. Such research would provide important information to physicians regarding how they should discuss treatment options to clients. In today's managed health-care environment, health providers often have less time with patients, yet are concerned with whether patients utilize the medical information that is provided. We believe that systematically providing base-rate and anecdotal information is likely to result in a more efficient procedure that helps patients to make the most medically efficacious decisions.

\section{REFERENCES}

Deber, R. B. (1994). Physicians in health care management: The patient-physician partnership: Changing roles and the desire for information. Canadian Medical Association, 151, 171176.

Greenfield, S., Kaplan, S., \& Ware, J. E. (1985). Expanding patient involvement in care: Effects on patient outcomes. Annals of Internal Medicine, 102, 520-528.

Kahneman, D., \& Tversky, A. (1973). On the psychology of prediction. Psychological Review, 80, 237-251.

Lerman, C. E., Brody, D. S., Caputo, G. C., Smith, D. G., Lazaro, C. G., \& Wolfson, H. G. (1990). Patients' perceived involvement in care scale: Relationship to attitudes about illness and medical care. Journal of General Internal Medicine, 5, 2933.

Mahler, H. I. M., \& Kulik, J. A. (1990). Preferences in health care involvement, perceived control and surgical recovery: A prospective study. Social Science Medicine, 31, 743-751.

Moyer, A., \& Solovey, P. (1998). Patient participation in treatment decision making and the psychological consequences of breast cancer surgery. Women's Health: Research on Gender, Behavior, and Policy, 4, 103-116.

Raghunathan, R., \& Pham, M. (1999). All negative moods are notequal: Motivational influences of anxiety \& sadness on decision making. Organizational Behavior and the Human Decision Processes, 1, 56-77.

Redelmeier, D. A., Rozin, P., \& Kahneman, D. (1993). Understanding patient's decisions: Cognitive and emotional perspectives. JAMA, 270, 72-76.

Speedling, E. J., \& Rose, D. N. (1985). Building and effective doctorpatient relationship: From patient satisfaction to patient participation. Social Science Medicine, 21, 115-120.

Tversky, A., \& Kahneman, D. (1971). Belief in the law of small numbers. Psychological Bulletin, 6, 105-110. 